\title{
ON GENERALIZED INVERSES OF SINGULAR MATRIX PENCILS
}

\author{
KLAUS RÖBENACK，KURT REINSCHKE
}

\author{
Institute of Control Theory \\ Faculty of Electrical and Computer Engineering, Technische Universität Dresden, D-01062 Dresden, Germany \\ e-mail:\{klaus.roebenack, kurt.reinschke\}@tu-dresden.de
}

\begin{abstract}
Linear time-invariant networks are modelled by linear differential-algebraic equations with constant coefficients. These equations can be represented by a matrix pencil. Many publications on this subject are restricted to regular matrix pencils. In particular, the influence of the Weierstrass structure of a regular pencil on the poles of its inverse is well known. In this paper we investigate singular matrix pencils. The relations between the Kronecker structure of a singular matrix pencil and the multiplicity of poles at zero of the Moore-Penrose inverse and the Drazin inverse of the rational matrix are investigated. We present example networks whose circuit equations yield singular matrix pencils.
\end{abstract}

Keywords: matrix pencils, Kronecker indices, Moore-Penrose inverse, Drazin inverse, linear networks.

\section{Introduction}

Various physical systems, especially electrical networks, can be modelled by differential-algebraic equations (DAEs),

$$
E \dot{x}(t)=A x(t)+B u(t), \quad x(0)=x_{0} .
$$

In the case of a regular matrix pencil $(E, A)$, the numerical properties of (1) are strongly influenced by the rational matrix $(E-h A)^{-1}$. For instance, if we apply the backward Euler method with a step size $h$ to (1), we obtain the recurrence relation

$$
\begin{aligned}
x\left(t_{n}\right)= & (E-h A)^{-1} E x\left(t_{n-1}\right) \\
& +(E-h A)^{-1} B u\left(t_{n}\right) .
\end{aligned}
$$

Analytical and numerical properties of (1) and (2) depend essentially on the index of the matrix pencil $(E, A)$ (Sincovec et al., 1981; Sannuti, 1981; Gear and Petzold, 1982; 1984; Hairer et al., 1989; Riaza, 2004). As for (2), the asymptotic behaviour of $(E-h A)^{-1}$ for $h \rightarrow 0$ is determined by the index.

In the case of a singular matrix pencil $(E, A)$, the inverse of $E-h A$ has to be replaced by a suitable generalized inverse $(E-h A)^{-}$, (Rao and Mitra, 1971; Boullion and Odell, 1971; Ben-Israel and Greville, 1974). In this paper, we will investigate the qualitative behaviour of generalized inverses of $E-h A$ for $h \rightarrow 0$. Although one would hardly use the corresponding generalization of (2) for a numerical solution of (1), these asymptotic properties are directly related to important system and control theoretical problems. To see this, we consider the Laplace-transformed form of (1), i.e.,

$$
(s E-A) X(s)=E x_{0}+B U(s) .
$$

If $s E-A$ is regular, the input-output behaviour of (3) can be described by the transfer function

$$
T(s)=C(s E-A)^{-1} B .
$$

If $s E-A$ is singular, we can replace the inverse occurring in (4) by a generalized inverse and obtain a generalized transfer function (Dziurla and Newcomb, 1987; Hou and Müller, 1992; Hou, 1995; Hou et al., 1997). For a singular matrix pencil, the system (1) might not be solvable, nor have a unique solution. Note that most publications on linear DAEs are restricted to the case of regular pencils, (e.g., Griepentrog and März, 1986; Brenan et al., 1996). Some results on singular pencils can be found in the works of Kronecker (1890), Gantmacher (1959), van Dooren (1981), Pandolfi (1981), Kublanovskaya (1983), or Röbenack and Reinschke (1998; 2000). In particular, the numerical solution of possibly singular DAEs is addressed by Kunkel et al. (1997), Kunkel and Mehrmann (2006), and Karcanias (1987). Even if the original pencil is regular, several control problems result in singular pencils (Kunkel and Mehrmann, 1990; Mehrmann, 1991). A further reason to study DAEs with singular pencil $(E, A)$ 
is the fact that the property of regularity is not feedback invariant (Özcaldiran and Lewis, 1990).

All standard circuits such as classical oscillators and amplifiers result in regular DAE models (Reinschke and Schwarz, 1976; Günther and Feldmann, 1999a; 1999b; Riaza 2008). Singular circuit equations or equations with higher index usually do not occur if the circuit is designed by hand. The above mentioned singularities are often the result of over-idealized modelling. Today, electronic circuits are usually designed with computer-aided design software. There, the interconnection of over-idealized subcircuits may result in a higher index or singular DAEs. An application where singular circuit equations occur is analogue fault detection (Straube et al., 2001). There, one simulates the response of a circuit to injected faults. Typical faults are short-circuits and disconnections, which are idealized models of physically possible faults. The faulty network is simulated and not checked manually. There, singularities indeed occur and may result in a wrong response of the circuit simulator.

Moreover, the kernel of the numerical solution of nonlinear DAEs is the solution of a linear system (Gear, 1971; Brenan et al., 1996). Singularities and certain bifurcations of nonlinear DAEs often result in a higher index or singular pencils of the associated linearized DAEs (Reißig, 1996; Reißig and Boche, 2003). These problems occur in power systems (Kwatny et al., 1995; Ayasun et al., 2004; Marszalek and Trzaska, 2005). Additionally, circuit equations of large scale systems may analytically be regular, but ill-conditioned matrices could result in a system which is singular from a numerical point of view (e.g., Bandler and Zhang, 1986).

Section 2 contains definitions and notations used in this paper. Moreover, this section gives some network examples that result in a higher index, as well as singular matrix pencils with arbitrarily large Kronecker indices. In addition, we recall an important result on the poles of $(E-h A)^{-1}$ at $h=0$ for the regular matrix pencil case.

We provide extensions of the regular case to singular matrix pencils in connection with generalized inverses. The Moore-Penrose inverse will be considered in Section 3 The Drazin inverse case will be investigated in Section 4 Conclusion are provided in Section 5

\section{Matrix pencils and index}

2.1. Weierstrass and Kronecker structure. In this section we introduce some definitions used in the paper. Let $M \in \mathbb{F}^{n \times n}$ denote an $n \times n$-matrix over a field $\mathbb{F}$. Then there exists a regular matrix $T \in G L(n, \mathbb{F})$ such

\footnotetext{
${ }^{1}$ The property regular of the matrix $G$ is synonymous to nonsingular, i.e., $T$ is square and $\operatorname{det} T \neq 0$. The regular $n \times n$-matrices over a field $\mathbb{F}$ form the general linear group $G L(n, \mathbb{F})$.
}

that

$$
T M T^{-1}=\left[\begin{array}{cc}
G & 0 \\
0 & N
\end{array}\right],
$$

where $G$ is a regular matrix and $N=\operatorname{diag}\left(N_{1}, \ldots, N_{d}\right)$ is a nilpotent block-diagonal matrix consisting of $\nu_{i} \times \nu_{i}$ dimensional Jordan blocks associated with the eigenvalue zero:

$$
N_{i}=\left(\begin{array}{cccc}
0 & 1 & \cdots & 0 \\
\vdots & \ddots & \ddots & \vdots \\
\vdots & & \ddots & 1 \\
0 & \cdots & \cdots & 0
\end{array}\right) \in \mathbb{R}^{\nu_{i} \times \nu_{i}}
$$

The index of the matrix $M$ is defined as the size of the greatest Jordan block $N_{i}$, i.e., ind $(M)=$ $\max \left\{\nu_{1}, \ldots, \nu_{d}, 0\right\}$. The index of a regular matrix is zero. In general, the index of a square matrix can be computed as follows:

$$
\operatorname{ind}(M)=\min \left\{i \in \mathbb{N}: \operatorname{rank} M^{i}=\operatorname{rank} M^{i+1}\right\},
$$

where $\mathbb{N}$ denotes the set of nonnegative integers. In accordance with our definition and Eqn. (6), the index of a $0 \times 0$-matrix is zero due to the absence of Jordan blocks.

Let $E, A \in \mathbb{R}^{l \times n}$. A matrix pencil2 $(E, A)$ is said to be regular if $l=n$ and $\operatorname{det}(E-h A) \not \equiv 0$. Otherwise, the matrix pencil is called singular. Each regular matrix pencil can be transformed into the Weierstrass canonical form (Weierstrass, 1868; Gantmacher, 1959),

$$
P(E-h A) Q=\left[\begin{array}{cc}
I-h W & 0 \\
0 & N-h I
\end{array}\right],
$$

with $P, Q \in G L(n, \mathbb{R})$. The matrix $W$ is a square matrix, the identity matrix is denoted by $I$, and the matrix $N$ is a nilpotent matrix. The index of a regular matrix pencil $(E, A)$ is defined as the index of the underlying matrix $N$ :

$$
\operatorname{ind}(E, A)=\operatorname{ind}(N) \text {. }
$$

The index of a regular matrix pencil is zero if and only if $E$ is regular. This means that the matrix $N$ has dimension $0 \times 0$, i.e., the block $N-h I$ does not occur in (7).

Using the concept of the normal form, one can obtain an expression for the index of the matrix pencil in terms of the original system matrices. Let $h \in \mathbb{C}$ and $\operatorname{det}(E-$ $h A) \neq 0$. Then we have

$$
\operatorname{ind}(E, A)=\operatorname{ind}\left((E-h A)^{-1} E\right),
$$

(see Griepentrog and März, 1986, Appendix A). As for regular matrix pencils, the condition $\operatorname{det}(E-h A) \neq 0$

\footnotetext{
${ }^{2}$ A matrix pencil of $E$ and $A$ is often denoted by $s E-A$ or $E-h A$, i.e., as a one-parametric subset of the space of matrices. In order to distinguish these matrix pencils from polynomial or rational matrices, we denote a pencil as an ordered pair $(E, A)$.
} 
holds in the whole of $\mathbb{C}$ except on a finite set, or from a topological point of view, for all $h$ belonging to an open and dense subset of $\mathbb{C}$. This implies that ind $(E, A)=$ $\operatorname{ind}\left((E-h A)^{-1} E\right)$ remains true even if $h$ is not fixed at a particular value and the index $\operatorname{ind}\left((E-h A)^{-1} E\right)$ is computed over the field $\mathbb{R}(h)$ of rational functions in $h$ with real coefficients.

Example 1. The rational matrix

$$
E-h A=\left[\begin{array}{l|ll}
h & 0 & 0 \\
\hline 0 & h & 1 \\
0 & 0 & h
\end{array}\right]
$$

has an index $\operatorname{ind}(E-h A)=0$ because of its regularity, whereas the associated matrix pencil $(E, A)$ has an index $\operatorname{ind}(E, A)=2$ :

$$
\begin{aligned}
\operatorname{ind}(E, A) & =\operatorname{ind}\left((E-h A)^{-1} E\right) \\
& =\operatorname{ind}\left[\begin{array}{c|cc}
0 & 0 & 0 \\
\hline 0 & 0 & h^{-1} \\
0 & 0 & 0
\end{array}\right] \\
& =2 .
\end{aligned}
$$

Each singular matrix pencil $(E, A)$ can be transformed into the Kronecker canonical form 3 ,

$$
\begin{aligned}
& P(E-h A) Q \\
& \quad=\operatorname{diag}\left(E_{r}-h A_{r}, E_{\varepsilon}-h A_{\varepsilon}, E_{\eta}-h A_{\eta}\right)
\end{aligned}
$$

with $P \in G L(l, \mathbb{R})$ and $Q \in G L(n, \mathbb{R})$ (cf. Kronecker, 1890; Gantmacher, 1959). The matrix pencil $\left(E_{r}, A_{r}\right)$ is regular. The singular matrix pencils $E_{\varepsilon}-h A_{\varepsilon}=\operatorname{diag}\left(L_{\varepsilon_{1}}, \ldots, L_{\varepsilon_{\rho}}\right)$ and $E_{\eta}-h A_{\eta}=$ $\operatorname{diag}\left(L_{\eta_{1}}^{\top}, \ldots, L_{\eta_{\sigma}}^{\top}\right)$ consist of $\kappa \times(\kappa+1)$-dimensional pencils $L_{\kappa}$ of the form

$$
L_{\kappa}=\left[\begin{array}{cccc}
h & 1 & 0 & 0 \\
0 & \ddots & \ddots & 0 \\
0 & 0 & h & 1
\end{array}\right]
$$

The nonnegative integers $\varepsilon_{i}$ (resp. $\eta_{j}$ ) are called right (resp. left) Kronecker indices. We define the index of a singular matrix pencil $(E, A)$ as the index of the regular part: $\operatorname{ind}(E, A)=\operatorname{ind}\left(E_{r}, A_{r}\right)$. If the regular part $E_{r}-h A_{r}$ is not present in (10), the index of the singular matrix pencil is zero.

The nilpotent matrix $N$ in (7) or in the regular part of (10) consists of Jordan blocks of the sizes $\nu_{1}, \ldots, \nu_{d}$ associated with the (generalized) eigenvalues of $E-h A$ at $h=0$ or, equivalently, with the finite elementary divisors $h^{\nu_{1}}, \ldots, h^{\nu_{d}}$ of $E-h A$. In $s E-A=s\left(E-\frac{1}{s} A\right)$,

\footnotetext{
${ }^{3}$ Note that the Kronecker canonical form 10 is only unique up to a permutation of its diagonal blocks.
}

the numbers $\nu_{i}$ are the Jordan block sizes of eigenvalues at $s=\infty$. In other words, the infinite elementary divisors of $s E-A$ correspond to the finite elementary divisors at zero of the dual pencil $E-h A$ (Karcanias and Hayton, 1981; Hayton et al., 1988).

The Kronecker canonical form (10) can be used to state an explicit solution of an arbitrary DAE (1) (see Gantmacher, 1959, Section 12.7). Each finite eigenvalue $s_{i} \in \mathbb{C}$ of $s E-A$ corresponds to an explicit linear ordinary differential equation (ODE) with Euler-type solutions of the homogeneous part (e.g., $\mathrm{e}^{s_{i} t}$ ). The inhomogeneous solution results from an integration (convolution) of the input signal $u$. Contrary to that, the infinite eigenvalues correspond to implicit ODEs having impulse solutions (Müller, 2005), where the input signal might be differentiated. In the time domain, each $\nu_{i} \times \nu_{i}$ Jordan block $N_{i}$ can be interpreted as a chain of $\nu_{i}$ successive differentiators. The index of the matrix pencil is the maximum length of such a chain of differentiators. The $L_{\varepsilon_{i}}$ and $L_{\eta_{j}}^{\top}$ blocks of the singular part correspond to underdetermined and overdetermined ODEs, respectively.

The numerical computation of the Kronecker canonical form 10 is not recommended because the transformation matrices $P$ and $Q$ can become ill conditioned. This problem can be circumvented using the quasi Kronecker canonical form,

$$
\begin{aligned}
\widetilde{P} & (E-h A) \widetilde{Q} \\
& =\left(\begin{array}{ccc}
\widetilde{E}_{\varepsilon}-h \widetilde{A}_{\varepsilon} & * & * \\
0 & \widetilde{E}_{r}-h \widetilde{A}_{r} & * \\
0 & 0 & \widetilde{E}_{\eta}-h \widetilde{A}_{\eta}
\end{array}\right),
\end{aligned}
$$

involving orthogonal matrices $\widetilde{P}$ and $\widetilde{Q}$ (see Wilkinson, 1979; Beelen and van Dooren, 1988; Demmel and Kågström, 1993). The matrix pencils $\widetilde{E}_{\varepsilon}-h \widetilde{A}_{\varepsilon}, \widetilde{E}_{r}-h \widetilde{A}_{r}$ and $\widetilde{E}_{\eta}-h \widetilde{A}_{\eta}$ are equivalent to the pencils $E_{\varepsilon}-h A_{\varepsilon}, E_{r}-$ $h A_{r}$ and $E_{\eta}-h A_{\eta}$, respectively, of the Kronecker canonical form 10). In particular, we have ind $\left(\widetilde{E}_{r}, \widetilde{A}_{r}\right)=$ $\operatorname{ind}\left(E_{r}, A_{r}\right)=\operatorname{ind}(E, A)$

Alternatively to the Weierstrass/Kronecker approach, one can analyze the elementary divisor structure at infinity using the Smith-MacMillan form (Vardulakis et al., 1982). In the general case of a rational matrix $F(s) \in$ $\mathbb{R}(s)^{l \times n}$ with $\varrho=\operatorname{rank} F(s)$ over the field $\mathbb{R}(s)$, there exist biproper matrices $4(s) \in \mathbb{R}(s)^{l \times l}$ and $Z(s) \in$ $\mathbb{R}(s)^{n \times n}$ such that

$$
\begin{aligned}
& Y(s) F(s) Z(s) \\
& =\operatorname{diag}\left(s^{q_{1}}, \ldots, s^{q_{\pi}}, s^{-\hat{q}_{\pi+1}}, \ldots, s^{-\hat{q}_{\varrho}}, 0_{(l-\varrho) \times(n-\varrho)}\right) \\
& =: S_{F(s)}^{\infty}(s),
\end{aligned}
$$

\footnotetext{
${ }^{4}$ A rational function is called proper if the numerator degree does not exceed the denominator degree. A square rational matrix with proper entries is called biproper if both the determinant and its inverse are proper functions, i.e., biproper matrices are units in the ring of proper matrices.
} 
where $q_{1} \geq \cdots \geq q_{\pi}>0$ and $0 \leq \hat{q}_{\pi+1} \leq \cdots \leq$ $\hat{q}_{\varrho}$. We say that $F(s)$ has $\pi$ poles at infinity, each one of order $q_{i}$. In addition, assume that $\tau \geq \pi$ is a number such that $\hat{q}_{\pi+1}=\cdots=\hat{q}_{\tau}=0$ and $\hat{q}_{\tau+1}, \ldots, \hat{q}_{\varrho}>0$. Then, we say that $F(s)$ has $(\varrho-\tau)$ zeros at infinity, each of order $\hat{q}_{j}$ with $j=\tau+1, \ldots, \varrho$.

Now, we consider the special case of a matrix pencil $s E-A$ with $E, A \in \mathbb{R}^{l \times n}, \pi=\operatorname{rank} E$, and $\varrho=$ $\operatorname{rank}(s E-A)$ over $\mathbb{R}(s)$. The construction of the SmithMacMillan form (12) results in a natural ordering of decreasing degrees of potencies. In order to be consistent with (12), we assume an increasing order of the Jordan block sizes $\nu_{1}=\cdots=\nu_{\varsigma}=1$ and $1<\nu_{\varsigma+1} \leq \cdots \leq \nu_{d}$, where $\varsigma=\tau-\pi$. The Smith-MacMillan form of $s E-A$ at infinity reads as

$$
\begin{aligned}
& S_{s E-A}^{\infty}(s) \\
& =Y(s)(s E-A) Z(s) \\
& =\operatorname{diag}\left(s I_{\pi}, s^{-\nu_{1}+1}, \ldots, s^{-\nu_{d}+1}, 0_{(l-\varrho) \times(n-\varrho)}\right) \\
& =\operatorname{diag}\left(s I_{\pi}, I_{\varsigma}, s^{-\nu_{\varsigma}+1}, \ldots, s^{-\nu_{d}+1}, 0_{(l-\varrho) \times(n-\varrho)}\right),
\end{aligned}
$$

with $\pi$ first order poles at $s=\infty$ (see Vardulakis and Karcanias, 1983, Proposition 2). The $d=\pi-\varrho$ Jordan blocks of $s E-A$ at infinity result in $d-\varsigma$ zeros at infinity of order $\hat{q}_{\tau+1}=\nu_{\varsigma+1}-1, \ldots, \hat{q}_{\varrho}=\nu_{d}-1$. If $s E-$ $A$ has at least one zero at infinity, we have ind $(E, A)=$ $\max \left\{\nu_{1}, \ldots \nu_{d}\right\}=\max \left\{\hat{q}_{\tau+1}+1, \ldots, \hat{q}_{\varrho}+1\right\}=\hat{q}_{\varrho}+1$, i.e., the index of $(E, A)$ is the greatest order of a zero of $s E-A$ at infinity increased by one. In the case of a regular matrix pencil, the zero diagonal block is omitted.

Example 2. Consider the matrix pencil $(E, A)$ with the matrices

$$
E=\left[\begin{array}{ccccc}
e_{11} & 0 & 0 & 0 & 0 \\
0 & 0 & 0 & 0 & 0 \\
0 & 0 & 0 & e_{34} & 0 \\
0 & 0 & 0 & 0 & 0 \\
0 & 0 & e_{53} & 0 & 0
\end{array}\right]
$$

and

$$
A=\left[\begin{array}{ccccc}
a_{11} & a_{12} & 0 & 0 & 0 \\
0 & 0 & a_{23} & 0 & 0 \\
0 & 0 & 0 & 0 & a_{35} \\
0 & 0 & 0 & 0 & 0 \\
0 & a_{52} & 0 & 0 & 0
\end{array}\right]
$$

The matrix pencil $(E, A)$ has the following Kronecker canonical form:

$$
P(E-h A) Q=\left[\begin{array}{c|cc|cc}
1-h \frac{a_{11}}{e_{11}} & 0 & 0 & 0 & 0 \\
\hline 0 & h & 1 & 0 & 0 \\
0 & 0 & h & 0 & 0 \\
\hline 0 & 0 & 0 & h & 1 \\
\hline 0 & 0 & 0 & 0 & 0
\end{array}\right] .
$$

The regular block has a $2 \times 2$-dimensional Jordan block associated with the eigenvalue zero, i.e., ind $(E, A)=$ 2 . The singular part consists of an $1 \times 2$-dimensional $L_{1}$-block and an $1 \times 0$-dimensional $L_{0}^{\top}$-block. Hence, the Kronecker indices are $\varepsilon_{1}=1$ and $\eta_{1}=0$. On the other hand, if we consider $E-h A$ a rational matrix over $\mathbb{R}(h)$, we have $\operatorname{rank}(E-h A)=\operatorname{rank}(E-h A)^{2}$, and therefore ind $(E-h A)=1$. Considering the pair $(E, A)$ as a rational matrix $s E-A$, we obtain the Smith-MacMillan form (13) at $s=\infty$ as $S_{s E-A}^{\infty}(s)=\operatorname{diag}(s, s, s, 1 / s, 0)$. In particular, we have a first order zero at $s=\infty$.

2.2. Network examples. We consider linear timeinvariant networks containing resistors, capacitors, inductors, operational amplifiers, as well as independent voltage and current sources. Assume that the network consists of $b$ branches. Let $p \in \mathbb{R}^{r}$ denote the vector containing all parameters of the network, i.e., resistances, capacitances, and inductances. The circuit equations are given by

$$
E(p) \dot{x}(t)=A(p) x(t)+B u(t),
$$

where $E$ and $A$ are parameter dependent $2 b \times 2 b$-matrices, $x$ denotes the $2 b$-dimensional vector,

$$
x=\left(v_{1}, \ldots, v_{b}, i_{1}, \ldots, i_{b}\right)^{\top},
$$

of the branch voltages $v_{1}, \ldots, v_{b}$ and the branch currents $i_{1}, \ldots, i_{b}$, and $u$ denotes the vector of the independent sources. The matrix $B$ describes which independent source influences which equation.

Neglecting the initial values, the Laplacetransformed version of (14) has the form

$$
\left(\begin{array}{c}
K \\
M_{0}(p)+s M_{1}(p)
\end{array}\right)\left(\begin{array}{c}
V_{1}(s) \\
\vdots \\
V_{b}(s) \\
I_{1}(s) \\
\vdots \\
I_{b}(s)
\end{array}\right)=B U(s)
$$

with the complex variable $s$. The Laplace-transformed signals are written in capital letters. Here, $K$ denotes the incidence matrices used to formulate Kirchhoff's voltage and current laws. The matrices $M_{0}$ and $M_{1}$ symbolize the network element relations. In particular, an independent voltage source $u_{i}$ represented by a branch $z$ has the network element relation

$$
\left(\begin{array}{ll}
1 & 0
\end{array}\right)\left(\begin{array}{c}
v_{z} \\
i_{z}
\end{array}\right)=u_{i}(t),
$$

and, in the case of an independent current source $u_{j}$, we have

$$
\left(\begin{array}{ll}
0 & 1
\end{array}\right)\left(\begin{array}{c}
v_{z} \\
i_{z}
\end{array}\right)=u_{j}(t)
$$


The operational amplifiers are assumed to be ideal, i.e., for the input branch $z_{1}$, both the branch voltage $v_{z_{1}}=0$ and the branch current $i_{z_{1}}=0$, as well as the branch voltage $v_{z_{2}}$ and the branch current $i_{z_{2}}$ of the output branch $z_{2}$ are determined by the remaining network elements (see Fig. 11). Overall, the contribution of such ideal operational amplifiers to the circuit equations (14) can be written as

$$
\left(\begin{array}{cccc}
1 & 0 & 0 & 0 \\
0 & 0 & 1 & 0
\end{array}\right)\left(\begin{array}{l}
v_{z_{1}} \\
v_{z_{2}} \\
i_{z_{1}} \\
i_{z_{2}}
\end{array}\right)=\left(\begin{array}{l}
0 \\
0
\end{array}\right)
$$

On the level of the network graph, ideal operational amplifiers are usually depicted as pairs of nullators and norators, where the nullator is the input branch and the norator the output branch (Davies, 1966; Fosséprez, 1992).

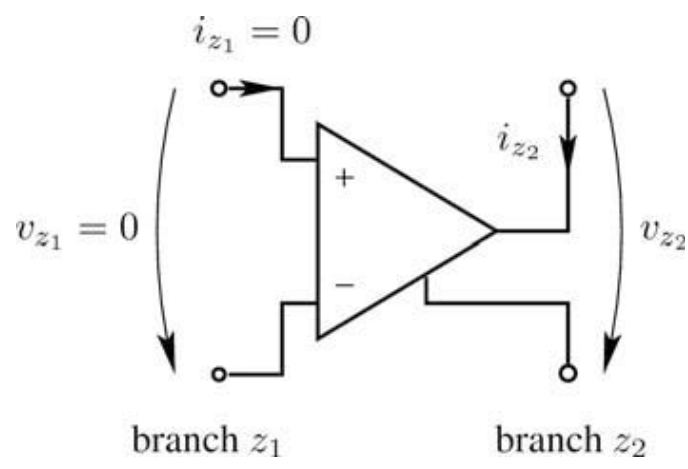

Fig. 1. Modelling of an operational amplifier.

The matrix pencil properties of the generalized inverses considered in Sections 3 and 4 depend essentially on the nilpotency index of the regular part and the Kronecker indices of the singular part. Most networks occurring in practical applications have network equations with regular matrix pencils of index one. However, various examples of a regular higher index as well as singular DAEs can be found in the literature (Fosséprez, 1992; Dziurla and Newcomb, 1989; Reißig, 1998; Röbenack, 1999; Tischendorf, 1996; 1999, Estévez Schwarz and Tischendorf, 2000; Riaza, 2006). These structures are often a result of overly simplifying assumptions. We will show with examples that these indices can be arbitrarily large, even for a network with only one active element.

When we consider network properties (e.g., singularity, index, Kronecker indices), we mean generic properties of the matrix pencils of the associated equation (14), i.e., properties which hold on an open and dense subset of the parameter space $\mathbb{R}^{r}$. Note that many (generic) network properties can be checked by inspecting the network graph. Results related to the network equations (14) can be found in the works of Hasler (1986), Fosséprez (1992) or Reißig (1999; 1998).
In the case of regular pencils, the generic index plays an important role. An example network with an arbitrarily large index is given by Reißig and Feldmann (1996), see Fig. 2. With $k$ inductors and $k-1$ capacitors, the network has $b=2 k+1$ branches. The associated circuit equations (14) have the dimension $n=4 k+2$. The underlying matrix pencil is generically regular. The Weierstrass canonical form (7) reads as

$$
P(E-h A) Q=\operatorname{diag}(N_{1}-h I, \underbrace{-h, \ldots,-h}_{2 k+2}),
$$

where $N_{1}$ is a nilpotent index $2 k$ matrix.

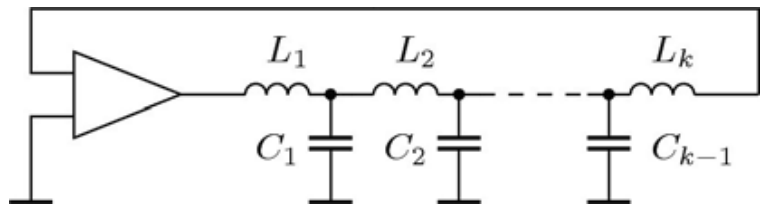

Fig. 2. Network with an arbitrarily large index (Reißig and Feldmann, 1996).

Now, we consider networks with singular circuit equations. Each network shown in Fig. 3 consists of $b=2 k+2$ branches. Hence, the vector $x$ of (14) has the dimension $n=4 k+4$. In both cases, the generic rank of the associated matrix pencil $E-h A$ is $n-1$ over the field $\mathbb{R}(h)$, which confirms that the pencils are indeed singular. The matrix pencil of the network shown in Fig. 3(a) has the Kronecker normal form,

$$
P(E-h A) Q=\operatorname{diag}(\underbrace{-h, \ldots,-h}_{2 k+4}, L_{2 k-1}, L_{0}^{\top}),
$$

whereas the network shown in Fig. 3 b) has the normal form,

$$
P(E-h A) Q=\operatorname{diag}(\underbrace{-h, \ldots,-h}_{2 k+4}, L_{0}, L_{2 k-1}^{\top}) .
$$

In the case of the first class of networks we have an arbitrarily large right Kronecker index $2 k-1$, and the second class of networks has an arbitrarily large left Kronecker index $2 k-1$

2.3. Pole multiplicity of inverses of regular matrix pencils. We remind the reader about the following relation between the index ind $(E, A)$ and the rational matrix $(E-h A)^{-1}$. Let $(E, A)$ be regular with $\operatorname{ind}(E, A)=k$. Using the Weierstrass canonical form (7), we obtain

$$
\begin{aligned}
(E & -h A)^{-1} \\
& =Q\left[\begin{array}{cc}
(I-h W)^{-1} & 0 \\
0 & (N-h I)^{-1}
\end{array}\right] P \\
& =Q\left[\begin{array}{cc}
(I-h W)^{-1} & 0 \\
0 & -\frac{1}{h} \sum_{l=0}^{k-1} \frac{N^{l}}{h^{l}}
\end{array}\right] P .
\end{aligned}
$$




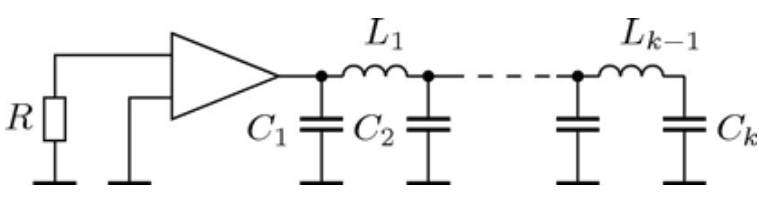

(a)

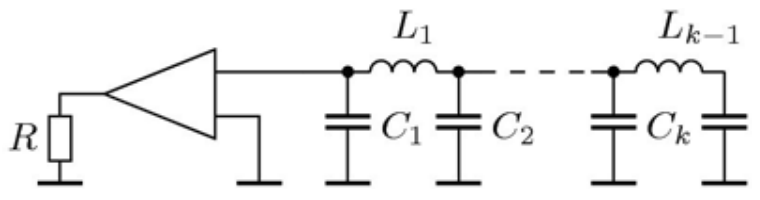

(b)

Fig. 3. Networks resulting in singular matrix pencils: with arbitrarily large right Kronecker indices (a), with arbitrary large left Kronecker indices (b).

The result is stated in the following theorem.

Theorem 1. (Brenan et al., 1996, Theorem 2.3.4) Let $(E, A)$ be regular with $k=\operatorname{ind}(E, A)$. Then the maximum multiplicity of a pole at $h=0$ of $(E-h A)^{-1}$ is equal to $k$.

Theorem 1 only states the maximum multiplicity, which reflects qualitative properties of the associated DAE (1). Let the nilpotent matrix $N$ in (7) have Jordan blocks of the sizes $\nu_{1} \leq \nu_{2} \leq \ldots \leq \nu_{d}$. Then $(E-h A)^{-1}$ has poles of order $\nu_{1}, \nu_{2}, \ldots, \nu_{d}$ at $h=0$, see (16). In a similar manner, from (13) we conclude that $(s E-A)^{-1}$ has the Smith-MacMillan form

$$
S_{(s E-A)^{-1}}^{\infty}(s)=\operatorname{diag}\left(s^{\nu_{d}-1}, \ldots, s^{\nu_{2}-1}, s^{\nu_{1}-1}, s^{-1} I_{\pi}\right) .
$$

Every $\nu_{i}>1$ corresponds to a pole of order $\nu_{i}-1$ at $s=\infty$.

To treat the singular matrix pencil case, we introduce a generalized inverse $M^{-}$of $M$ defined by $M=$ $M M^{-} M$ (cf. Rao and Mitra, 1971; Boullion and Odell, 1971; Ben-Israel and Greville, 1974). The next example shows that Theorem 1 cannot be extended to the singular matrix pencil case for all generalized inverses.

Example 3. Consider the matrix pencil $(E, A)$ of Example 1 and

$$
\hat{E}-h \hat{A}=\left[\begin{array}{c|cc}
h & 0 & 0 \\
\hline 0 & 0 & 0 \\
0 & 0 & 0
\end{array}\right],
$$

with $\operatorname{ind}(\hat{E}, \hat{A})=\operatorname{ind}\left(\hat{E}_{r}, \hat{A}_{r}\right)=1$. The matrix

$$
\begin{aligned}
(E-h A)^{-1} & =\left[\begin{array}{c|cc}
h^{-1} & 0 & 0 \\
\hline 0 & h^{-1} & -h^{-2} \\
0 & 0 & h^{-1}
\end{array}\right] \\
& =(\hat{E}-h \hat{A})^{-}
\end{aligned}
$$

is a generalized inverse of $\hat{E}-h \hat{A}$. Obviously, the matrix $(E-h A)^{-1}$ has a second order pole at $h=0$. Equivalently, the rational matrix $(s E-A)^{-1}$ has the SmithMacMillan form $S_{(s E-A)^{-1}}^{\infty}(s)=\operatorname{diag}(s, 1,1 / s)$ and therefore a first order pole at $s=\infty$.

We proceed to show that a generalization of Theorem 1 is possible if we consider the Moore-Penrose inverse.

\section{Moore-Penrose inverse case}

For a matrix $M \in \mathbb{F}^{l \times n}$ over the field $\mathbb{F}=\mathbb{C}$, the Moore-Penrose inverse $M^{+} \in \mathbb{F}^{n \times l}$ is uniquely determined by the following equations (Penrose, 1955): $M M^{+} M=M, M^{+} M M^{+}=M^{+},\left(M M^{+}\right)^{*}=$ $M M^{+},\left(M^{+} M\right)^{*}=M^{+} M$, where $M^{*} \in \mathbb{F}^{n \times l}$ denotes the conjugate transpose of $M$. We will use these equations to define a Moore-Penrose inverse over the field $\mathbb{F}=\mathbb{R}(h)$ with $h \in \mathbb{R}$ or $h \in \mathbb{C}$. Note that for rational functions the conjugate value $\bar{h}$ of $h$ may differently be defined as the standard conjugate value in $\mathbb{C}$. This is due to a different definition of a conjugate matrix for dynamical systems, i.e., if the rational matrix is used as a transfer function, (see Varga, 1998; 2001).

In the proof of Theorem 1 we used the Weierstrass canonical form and the identity $(P(E-h A) Q)^{-1}=$ $Q^{-1}(E-h A)^{-1} P^{-1}$. Unfortunately, we cannot use the same direct approach to prove Theorem 2 because $(P(E-h A) Q)^{+} \neq Q^{+}(E-h A)^{+} P^{+}$in general for singular matrix pencils. As a preparation for the MoorePenrose inverse case we also would like to remind the reader about some facts concerning singular value decomposition. For a real-analytic matrix valued function $M:[a, b] \rightarrow \mathbb{R}^{l \times n}$, an analytic singular value decomposition is a factorization

$$
M(t)=U(t) \cdot \Sigma(t) \cdot V^{\top}(t), \quad t \in[a, b],
$$

where $U:[a, b] \rightarrow G L(l, \mathbb{R})$ and $V:[a, b] \rightarrow G L(n, \mathbb{R})$ are orthogonal, $\Sigma:[a, b] \rightarrow \mathbb{R}^{l \times n}$ is a diagonal matrix, and $U(\cdot), \Sigma(\cdot)$ and $V(\cdot)$ are analytic. We need the following result.

Proposition 1. (Bunse-Gerstner et al., 1991) Let $M$ : $[a, b] \rightarrow \mathbb{R}^{l \times n}$ be analytic. Then there exists an analytic singular value decomposition (17) on the interval $[a, b]$.

The following theorem extends Theorem 1 to the Moore-Penrose inverse case.

Theorem 2. Let $E, A \in \mathbb{R}^{l \times n}$ and $k=\operatorname{ind}(E, A)$. Then the maximum multiplicity of a pole at $h=0$ of $(E-h A)^{+}$ is equal to $k$.

Proof. For $h=0$ we have $h=\bar{h}$. This implies that the multiplicity of a pole over $\mathbb{R}(h)$ is the same for $h \in \mathbb{R}$ and $h \in \mathbb{C}$. Without loss of generality we use $h \in \mathbb{R}$ in 
the proof. For $E, A \in \mathbb{R}^{l \times n}$, the pencil $E-h A$ can be transformed into the Kronecker canonical form (10). The block $E_{r}-h A_{r}$ is regular over $\mathbb{R}(h)$. For all $h \in \mathbb{R}$, the singular blocks $E_{\varepsilon}-h A_{\varepsilon}$ and $E_{\eta}-h A_{\eta}$ have full row rank and full column rank, respectively. The matrix $E-h A$ can be written as the product of a column-regular matrix $G$ and a row-regular matrix $H$ (over the field $\mathbb{R}(h)$ ):

$$
\begin{aligned}
E-h A= & \underbrace{P^{-1}\left[\begin{array}{ccc}
I & 0 & 0 \\
0 & E_{\eta}-h A_{\eta} & 0 \\
0 & 0 & E_{r}-h A_{r}
\end{array}\right]}_{=: G} \\
& \times \underbrace{\left[\begin{array}{ccc}
E_{\varepsilon}-h A_{\varepsilon} & 0 & 0 \\
0 & I & 0 \\
0 & 0 & I
\end{array}\right] Q^{-1}}_{=: H} .
\end{aligned}
$$

Under these circumstances, we have $(E-h A)^{+}=$ $(G H)^{+}=H^{+} G^{+}$(Boullion and Odell, 1971, Section 2.1; Campbell and Meyer, 1979, Corollary 1.4.2). The matrix $H$ has constant rank for all $h \in \mathbb{R}$, in particular for all $h \in[-1,1]$. Clearly, the affine-linear mapping $h \mapsto H(h)$ is analytic. According to Propsition 11, there exists an analytic singular value decomposition $H(h)=U(h) \cdot \Sigma(h) \cdot V(h)^{\top}$ with $\Sigma(h)=$ $\operatorname{diag}\left(\sigma_{1}(h), \ldots, \sigma_{\rho}(h), 0, \ldots, 0\right)$ on the interval $[-1,1]$. Because the matrix $H$ has constant rank for all $h \in$ $[-1,1]$, the Moore-Penrose inverse $H(h)^{+}=V(h)$. $\Sigma(h)^{+} \cdot U(h)^{\top}$ has no poles at $h=0$.

The matrix $G$ can be written as the product of a column-regular matrix $J$ and a row-regular matrix $K$ :

$$
\begin{aligned}
G= & \underbrace{P^{-1}\left[\begin{array}{ccc}
I & 0 & 0 \\
0 & E_{\eta}-h A_{\eta} & 0 \\
0 & 0 & I
\end{array}\right]}_{=: J} \\
& \times \underbrace{\left[\begin{array}{ccc}
I & 0 & 0 \\
0 & I & 0 \\
0 & 0 & E_{r}-h A_{r}
\end{array}\right]}_{=: K} .
\end{aligned}
$$

We obtain $G^{+}=(J K)^{+}=K^{+} J^{+}$. The analytic matrix valued function $J$ has constant rank for all $h \in[-1,1]$. Hence, the Moore-Penrose inverse $J^{+}$has no poles at $h=0$. It remains to analyze the matrix $K$. Using (19), we obtain the following expression of the Moore-Penrose inverse of $K$ :

$$
\begin{aligned}
K^{+} & =\left[\begin{array}{ccc}
I & 0 & 0 \\
0 & I & 0 \\
0 & 0 & \left(E_{r}-h A_{r}\right)^{+}
\end{array}\right] \\
& =\left[\begin{array}{ccc}
I & 0 & 0 \\
0 & I & 0 \\
0 & 0 & \left(E_{r}-h A_{r}\right)^{-1}
\end{array}\right] \\
& =K^{-1} .
\end{aligned}
$$

The last expression indicates that $K$ is regular over the field $\mathbb{R}(h)$. Obviously, the poles of $K^{-1}$ at $h=0$ are exactly the poles of the regular part $\left(E_{r}-h A_{r}\right)^{-1}$ at $h=$ 0 . Here, we can apply Theorem 1 . Since ind $(E, A)=$ $\operatorname{ind}\left(E_{r}, A_{r}\right)$ (by definition), the maximum multiplicity of a pole at $h=0$ of the entries of $(E-h A)^{+}$is equal to the index $\operatorname{ind}(E, A)$.

Equation (9) can be generalized to the Moore-Penrose inverse case of a singular pencil as follows.

Proposition 2. Let $E, A \in \mathbb{R}^{l \times n}$ be singular and $k=$ $\operatorname{ind}(E, A)$. Then we have

$$
\max \{k, 1\} \leq \operatorname{ind}\left((E-h A)^{+} E\right) \leq k+1 .
$$

Proof. We calculate $(E-h A)^{+} E$ directly using the quasi Kronecker canonical form (11). Since $\widetilde{P}$ and $\widetilde{Q}$ are orthogonal, we obtain

$$
\begin{aligned}
& (E-h A)^{+} E \\
& \quad=\widetilde{Q}\left(\begin{array}{ccc}
\Delta_{\varepsilon}(h) & * & * \\
0 & \Delta_{r}(h) & * \\
0 & 0 & \Delta_{\eta}(h)
\end{array}\right) \widetilde{Q}^{-1},
\end{aligned}
$$

with the diagonal matrices

$$
\begin{aligned}
& \Delta_{\varepsilon}(h)=\left(\widetilde{E}_{\varepsilon}-h \widetilde{A}_{\varepsilon}\right)^{+} \widetilde{E}_{\varepsilon}, \\
& \Delta_{r}(h)=\left(\widetilde{E}_{r}-h \widetilde{A}_{r}\right)^{+} \widetilde{E}_{r}, \\
& \Delta_{\eta}(h)=\left(\widetilde{E}_{\eta}-h \widetilde{A}_{\eta}\right)^{+} \widetilde{E}_{\eta}
\end{aligned}
$$

due to the results of Campbell and Meyer (1979, Theorem 1.2.1 and Theorem 3.4.1).

Because $\widetilde{E}_{\eta}-h \widetilde{A}_{\eta}$ is column regular for all $h \in \mathbb{C}$, we have $\widetilde{E}_{\eta}^{+} \widetilde{E}_{\eta}=I$ and $\left(\widetilde{E}_{\eta}-h \widetilde{A}_{\eta}\right)^{+}\left(\widetilde{E}_{\eta}-h \widetilde{A}_{\eta}\right)=I$. Therefore, $\left(\widetilde{E}_{\eta}-h \widetilde{A}_{\eta}\right)+\widetilde{E}_{\eta}$ is regular over $\mathbb{R}(h)$, i.e., $\operatorname{ind}\left(\left(\widetilde{E}_{\eta}-h \widetilde{A}_{\eta}\right)+\widetilde{E}_{\eta}\right)=0$. Since $\widetilde{E}_{r}-h \widetilde{A}_{r}$ is regular by construction, we have $\left(\widetilde{E}_{r}-h \widetilde{A}_{r}\right)^{+}=\left(\widetilde{E}_{r}-\right.$ $\left.h \widetilde{A}_{r}\right)^{-1}$. This implies ind $\left(\left(\widetilde{E}_{r}-h \widetilde{A}_{r}\right)^{+} \widetilde{E}_{r}\right)=\operatorname{ind}\left(\left(\widetilde{E}_{r}-\right.\right.$ $\left.\left.h \widetilde{A}_{r}\right)^{-1} \widetilde{E}_{r}\right)=\operatorname{ind}(E, A)$ due to (9). In the trivial case of a matrix pencil $\widetilde{E}_{\varepsilon}-h \widetilde{A}_{\varepsilon}$ with zero rows, we have $\operatorname{ind}\left(\left(\widetilde{E}_{\varepsilon}-h \widetilde{A}_{\varepsilon}\right)+\widetilde{E}_{\varepsilon}\right)=0$. Otherwise, $\widetilde{E}_{\varepsilon}-h \widetilde{A}_{\varepsilon}$ is row regular for all $h \in \mathbb{C}$. Therefore, $\left(\widetilde{E}_{\varepsilon}-h \widetilde{A}_{\varepsilon}\right)+\left(\widetilde{E}_{\varepsilon}-h \widetilde{A}_{\varepsilon}\right)$ is an orthogonal projector. In particular, the projector has only 0 and 1 as eigenvalues, each of Jordan block size $1 \times 1$, i.e., $\operatorname{ind}\left(\left(\widetilde{E}_{\varepsilon}-h \widetilde{A}_{\varepsilon}\right)^{+}\left(\widetilde{E}_{\varepsilon}-h \widetilde{A}_{\varepsilon}\right)\right)=1$.

Due to the projector property and the row regularity of $\widetilde{E}_{\varepsilon}-h \widetilde{A}_{\varepsilon}$, we have $\operatorname{rank}\left(\left(\widetilde{E}_{\varepsilon}-h \widetilde{A}_{\varepsilon}\right)^{+}\left(\widetilde{E}_{\varepsilon}-h \widetilde{A}_{\varepsilon}\right)\right) \geq$ $\operatorname{rank}\left(\left(\widetilde{E}_{\varepsilon}-h \widetilde{A}_{\varepsilon}\right)^{+} \widetilde{E}_{\varepsilon}\right)$. Hence, the perturbed matrix $\left(\widetilde{E}_{\varepsilon}-h \widetilde{A}_{\varepsilon}\right)+\widetilde{E}_{\varepsilon}$ (locally with respect to $h$ ) will still have $1 \times 1$ Jordan blocks associated with the zero eigenvalues, i.e., over $\mathbb{R}(h)$ we have ind $\left(\left(\widetilde{E}_{\varepsilon}-h \widetilde{A}_{\varepsilon}\right)^{+} \widetilde{E}_{\varepsilon}\right)=1$. Having established the indices of the diagonal matrices (23) of (22), the bounds (21) for the index of the block diagonal matrix result from Meyer and Rose (1977, Theorem 2.1). 
Example 4. The matrix pencil of Example 2 has index $\operatorname{ind}(E, A)=2$. This can also be verified by investigating the Moore-Penrose inverse shown in Fig. 4. The maximum multiplicity of a pole at $h=0$ of $(E-h A)^{+}$is 2 . In addition, we have $\operatorname{ind}\left((E-h A)^{+} E\right)=2$. The rational matrix $(s E-A)^{+}$has the Smith-MacMillan form $S_{(s E-A)+}^{\infty}(s)=\operatorname{diag}\left(s, \frac{1}{s}, \frac{1}{s}, \frac{1}{s}, 0\right)$ with maximum multiplicity ind $(E, A)-1=1$ of poles at $s=\infty$.

\section{Drazin inverse case}

For a square matrix $M \in \mathbb{F}^{n \times n}$ with the decomposition (5), the Drazin inverse $M^{D} \in \mathbb{F}^{n \times n}$ is given by

$$
M^{D}=T^{-1}\left[\begin{array}{cc}
G^{-1} & 0 \\
0 & 0
\end{array}\right] T .
$$

Whereas the Moore-Penrose inverse can be computed by inverting the non-vanishing singular values, the Drazin inverse can be computed via the inversion of the nonvanishing eigenvalues. The Drazin inverse $M^{D}$ of $M$ is a generalized inverse (in the sense of Section 2.3 , i.e., $M=M M^{D} M$ ) if and only if $\operatorname{ind}(M) \leq 1$ (Campbell and Meyer, 1979, Theorem 7.2.4). If $M$ is the system matrix of a linear system of equations (as in the case of the network examples discussed in Section 2.2), the condition $\operatorname{ind}(M) \leq 1$ can always be achieved without changing the solution or the solvability of the system, e.g., by row reduction.

The decompositions (5) and (24) illustrate another characterization of the Drazin inverse (Campbell and Meyer, 1979, Definition 7.2.2): For $k=\operatorname{ind}(M)$, the vector space $\mathbb{F}^{n}$ can be written as a direct sum $\mathbb{F}^{n}=$ $\operatorname{im} M^{k} \oplus \operatorname{ker} M^{k}$, i.e., every vector $z \in \mathbb{F}^{n}$ has a unique representation $z=x+y$ with $x \in \operatorname{im} M^{k}$ and $y \in \operatorname{ker} M^{k}$. Let $\mathcal{M}: \mathbb{F}^{n} \rightarrow \mathbb{F}^{n}$ denote a linear map described by the matrix $M$ and let $\widetilde{\mathcal{M}}=\left.\mathcal{M}\right|_{\text {im } M^{k}}$ be the restriction of $\mathcal{M}$ to the subspace $\operatorname{im} M^{k}$. Since $\widetilde{\mathcal{M}}: \operatorname{im} M^{k} \rightarrow \operatorname{im} M^{k}$ is bijective, there exists the inverse map $\widetilde{\mathcal{M}}^{-1}$. The Drazin inverse map $\mathcal{M}^{D}$ is defined by $\mathcal{M}^{D} z=\widetilde{\mathcal{M}}^{-1} x$, and the Drazin inverse $M^{D}$ is the matrix associated with the map $\mathcal{M}^{D}$.

The next example shows that, in contrast to the Moore-Penrose inverse, the poles of $(E-h A)^{D}$ are not uniquely determined by the index of the matrix pencil.

Example 5. Both matrix pencils $\left(E_{1}, A_{1}\right)$ and $\left(E_{2}, A_{2}\right)$ have the same Kronecker canonical form with index $\operatorname{ind}\left(E_{i}, A_{i}\right)=2(i=1,2)$ :

$$
\begin{aligned}
& E_{1}-h A_{1}=\left[\begin{array}{ll|l}
h & 1 & 0 \\
0 & h & 0 \\
\hline 0 & 0 & 0
\end{array}\right], \\
& E_{2}-h A_{2}=\left[\begin{array}{l|ll}
0 & h & 1 \\
0 & 0 & h \\
\hline 0 & 0 & 0
\end{array}\right] .
\end{aligned}
$$

Whereas the Drazin inverse of the first pencil contains a second order pole at $h=0$, the Drazin inverse of the second matrix pencil is the zero matrix because the rational matrix $E_{2}-h A_{2}$ is nilpotent:

$$
\begin{aligned}
& \left(E_{1}-h A_{1}\right)^{D}=\left[\begin{array}{rr|r}
\frac{1}{h} & -\frac{1}{h^{2}} & 0 \\
0 & \frac{1}{h} & 0 \\
\hline 0 & 0 & 0
\end{array}\right], \\
& \left(E_{2}-h A_{2}\right)^{D}=\left[\begin{array}{lll}
0 & 0 & 0 \\
0 & 0 & 0 \\
0 & 0 & 0
\end{array}\right] .
\end{aligned}
$$

Similarly, both pencils have the same Smith-MacMillan form $S_{s E_{1}-A_{1}}^{\infty}(s)=S_{s E_{2}-A_{2}}^{\infty}(s)=\operatorname{diag}\left(s, \frac{1}{s}, 0\right)$ at $s=\infty$, but their Drazin inverses have different structures: $S_{\left(s E_{1}-A_{1}\right)^{D}}^{\infty}(s)=\operatorname{diag}\left(s, \frac{1}{s}, 0\right)$ and $S_{\left(s E_{2}-A_{2}\right)^{D}}^{\infty}(s)=$ $\operatorname{diag}(0,0,0)$.

Even when $\operatorname{ind}(E-h A)=1$, the poles of $(E-$ $h A)^{D}$ are not uniquely determined by the Kronecker structure of $(E, A)$. In the next case, each matrix pencil $\left(E_{3}, A_{3}\right)$ and $\left(E_{4}, A_{4}\right)$ only consists of one $L_{2}$ block together with one $L_{0}^{\top}$ block:

$$
\begin{aligned}
& E_{3}-h A_{3}=\left[\begin{array}{lll}
h & 1 & 0 \\
0 & h & 1 \\
\hline 0 & 0 & 0
\end{array}\right], \\
& E_{4}-h A_{4}=\left[\begin{array}{lll}
1 & h & 0 \\
0 & 1 & h \\
\hline 0 & 0 & 0
\end{array}\right] .
\end{aligned}
$$

Neither of the matrix pencils has a regular part. However, the rational matrix $\left(E_{3}-h A_{3}\right)^{D}$ has poles at $h=0$ and $\left(E_{4}-h A_{4}\right)^{D}$ has no poles.

$$
\begin{aligned}
& \left(E_{3}-h A_{3}\right)^{D}=\left[\begin{array}{rrr}
\frac{1}{h} & -\frac{1}{h^{2}} & -\frac{2}{h^{3}} \\
0 & \frac{1}{h} & \frac{1}{h^{2}} \\
\hline 0 & 0 & 0
\end{array}\right], \\
& \left(E_{4}-h A_{4}\right)^{D}=\left[\begin{array}{rrr}
1 & -h & -2 h \\
0 & 1 & h \\
\hline 0 & 0 & 0
\end{array}\right] .
\end{aligned}
$$

Likewise, both pencils have the same SmithMacMillan form $S_{s E_{3}-A_{3}}^{\infty}(s)=S_{s E_{4}-A_{4}}^{\infty}(s)=$ $\operatorname{diag}(s, s, 0)$ at $s=\infty$, but their Drazin inverses differ significantly: $S_{\left(s E_{3}-A_{3}\right) D}^{\infty}(s)=\operatorname{diag}\left(s^{2}, 1,0\right)$ and $S_{\left(s E_{4}-A_{4}\right)^{D}}^{\infty}(s)=\operatorname{diag}\left(\frac{1}{s}, \frac{1}{s}, 0\right)$.

The following theorem gives lower and upper bounds to the multiplicity of poles at $h=0$ of the rational matrix $(E-h A)^{D}$. We restrict ourselves to $\operatorname{ind}(E-h A)=$ 1 (over $\mathbb{R}(h)$ ), where the Drazin inverse is a generalized inverse.

Theorem 3. Let ind $(E-h A)=1$ and $k=\operatorname{ind}(E, A)$. Furthermore, let $\varepsilon_{i}$ and $\eta_{j}$ denote the right and left Kronecker indices, respectively. Then the maximum multiplicity $\delta$ of poles at $h=0$ in the entries of $(E-h A)^{D}$ is 


$$
\begin{aligned}
& (E-h A)^{+}=\left[\begin{array}{ccccc}
\frac{1}{e_{11}-a_{11} h} & \frac{a_{12} e_{53}}{a_{23} a_{52}\left(a_{11} h-e_{11}\right)} & 0 & 0 & \frac{a_{12}}{a_{52}\left(a_{11} h-e_{11}\right)} \\
0 & -\frac{e_{53}}{a_{23} a_{52} h^{2}} & 0 & 0 & -\frac{1}{a_{52} h} \\
0 & -\frac{1}{a_{23} h} & 0 & 0 & 0 \\
0 & 0 & \frac{e_{34}}{e_{34}^{2}+a_{35}^{2} h^{2}} & 0 & 0 \\
0 & 0 & -\frac{a_{35} h}{e_{34}^{2}+a_{35}^{2} h^{2}} & 0 & 0
\end{array}\right] \\
& (E-h A)^{D}=\left[\begin{array}{ccccc}
\frac{1}{e_{11}-h a_{11}} & \frac{a_{12} e_{53}}{h a_{23} a_{52}\left(h a_{11}-e_{11}\right)} & 0 & \frac{a_{12} e_{34}}{h a_{35} a_{52}\left(e_{11}-h a_{11}\right)} & \frac{a_{12}}{a_{52}\left(h a_{11}-e_{11}\right)} \\
0 & -\frac{e_{53}}{h^{2} a_{23} a_{52}} & 0 & \frac{e_{34}}{h^{2} a_{35} a_{52}} & -\frac{1}{h a_{52}} \\
0 & -\frac{1}{h a_{23}} & 0 & 0 & 0 \\
0 & 0 & 0 & 0 & 0 \\
0 & 0 & -\frac{1}{h a_{35}} & 0 & 0
\end{array}\right]
\end{aligned}
$$

Fig. 4. Generalized inverses of the matrix pencil from Example 2 used in Examples 4 and 6

bounded by the inequality

$$
k \leq \delta \leq \max _{i, j}\left\{k, \varepsilon_{i}+1, \eta_{j}+1\right\} .
$$

Proof. Let $M=E-h A, \operatorname{ind}(M)=1$, and $k=$ $\operatorname{ind}(E, A)$. To analyze the properties of the Drazin inverse $M^{D}$, we consider the endomorphism

$$
\mathcal{M}: \mathbb{R}^{n} \rightarrow \operatorname{im} M \subset \mathbb{R}^{l}: x \mapsto M x .
$$

The mapping $\mathcal{M}$ is surjective by definition. The subspace $\operatorname{im} M$ can be written as a direct sum,

$$
\begin{aligned}
\operatorname{im} M= & \operatorname{im}(E-h A) \\
= & P^{-1}\left[\operatorname{im}\left(E_{r}-h A_{r}\right) \oplus \operatorname{im} L_{\varepsilon_{1}} \oplus \cdots\right. \\
& \left.\cdots \oplus \operatorname{im} L_{\varepsilon_{\rho}} \oplus \operatorname{im} L_{\eta_{1}}^{\top} \oplus \cdots \oplus \operatorname{im} L_{\eta_{\sigma}}^{\top}\right] Q^{-1},
\end{aligned}
$$

associated with the block-diagonal representation (10) of $M$. In order to analyze the poles of $M^{D}$, we investigate the inverse mappings of $\mathcal{M}$ for each block. We start with the regular block $E_{r}-h A_{r}$. The inverse mapping of this block is characterized by $\left(E_{r}-h A_{r}\right)^{-1}$. According to Theorem 11 the maximum order of a pole of $\left(E_{r}-h A_{r}\right)^{-1}$ at $h=0$ is equal to the index $k$. This gives us a lower bound $k \leq \delta$.

Now, let us consider a singular block $L_{\varepsilon_{i}}$. We analyze the associated linear mapping $u \mapsto v=L_{\varepsilon_{i}} u$ with

$$
\left[\begin{array}{cccc}
h & 1 & & 0 \\
& \ddots & \ddots & \\
0 & & h & 1
\end{array}\right]\left[\begin{array}{c}
u_{1} \\
\vdots \\
u_{\varepsilon_{i}+1}
\end{array}\right]=\left[\begin{array}{c}
v_{1} \\
\vdots \\
v_{\varepsilon_{i}}
\end{array}\right]
$$

The solution of this system of linear equations is a one-dimensional linear manifold. With the additional constraint $\left(\alpha_{\varepsilon_{i}+1}+\beta_{\varepsilon_{i}+1} h, \alpha_{\varepsilon_{i}}+\beta_{\varepsilon_{i}} h, \ldots, \alpha_{1}+\beta_{1} h\right)$, we select one solution from this manifold. The matrix

$$
\begin{aligned}
& M_{0}+h M_{1} \\
& =\left[\begin{array}{cccc}
h & 1 & & 0 \\
& \ddots & \ddots & \\
0 & & h & 1 \\
\hline \alpha_{\varepsilon_{i}+1}+\beta_{\varepsilon_{i}+1} h & \cdots & \alpha_{2}+\beta_{2} h & \alpha_{1}+\beta_{1} h
\end{array}\right]
\end{aligned}
$$

of the resulting system should be regular and its inverse should have a pole at $h=0$ with maximum multiplicity. A determinant term $h^{\varepsilon_{i}+1}$ occurs only for $\beta_{1} \neq 0$. For this reason, we set $\beta_{1}=1$. The remaining terms of the determinant

$$
\begin{aligned}
\operatorname{det}\left(M_{0}+h M_{1}\right)= & \alpha_{\varepsilon_{i}+1}+\left(\beta_{\varepsilon_{i}+1}-\alpha_{\varepsilon_{i}}\right) h+\cdots \\
& +\left(\beta_{2}-\alpha_{1}\right) h^{\varepsilon_{i}}+h^{\varepsilon_{i}+1}
\end{aligned}
$$

cancel out each other if $\alpha_{\varepsilon_{i}+1}=0$ and $\beta_{\varepsilon_{i}+1}=\alpha_{\varepsilon_{i}}, \ldots$, $\beta_{2}=\alpha_{1}$. We obtain $N=M_{1}^{-1} M_{0}$ with

$$
N=\left[\begin{array}{cccc}
0 & 1 & & 0 \\
& \ddots & \ddots & \\
& & \ddots & 1 \\
0 & & & 0
\end{array}\right] .
$$

The matrix $N$ has index ind $(N)=\varepsilon_{i}+1$. The inverse of 
the matrix $M_{0}+h M_{1}$ has the following form:

$$
\begin{aligned}
\left(M_{0}+h M_{1}\right)^{-1} & =(h I+N)^{-1} M_{1}^{-1} \\
& =\left[\frac{1}{h} \sum_{i=0}^{\varepsilon_{i}} \frac{(-N)^{i}}{h^{i}}\right] M_{1}^{-1} .
\end{aligned}
$$

Due to this expression, the multiplicity of poles at $h=0$ of the inverse mapping is at most $\varepsilon_{i}+1$. Since $\left(M^{D}\right)^{\top}=$ $\left(M^{\top}\right)^{D}$, the same can be applied to every $L_{\eta_{j}}^{\top}$ block. Hence, the maximum multiplicity of a pole at $h=0$ of $(E-h A)^{D}$ induced by the singular part is less or equal to $\max _{i, j}\left\{\varepsilon_{i}+1, \eta_{j}+1\right\}$. This proves Eqn. (25).

The proof also reveals that when $\operatorname{ind}(E-h A)=1$, each nilpotent $\nu_{\ell} \times \nu_{\ell}$-Jordan block of the regular part $E_{r}-h A_{r}$ results in a pole of $(E-h A)^{D}$ at $h=0$ of order $\nu_{\ell}$. The occurrence of poles at $h=0$ induced by the Kronecker structure of the singular part depends essentially on the relation between the coordinates of the equivalence transformation (10) and the similarity transformation in (5) and (24). The Smith-MacMillan form (12) of $(s E-A)^{D}$ will have poles of order $\nu_{\ell}-1$ at $s=\infty$. There might be additional poles at $s=\infty$ resulting from the singular part with the maximum multiplicity $\max _{i, j}\left\{\varepsilon_{i}, \eta_{j}\right\}$.

Example 6. For the multiplicity of the poles at $h=0$ of the rational matrix $(E-h A)^{D}$ of Example 2 we get the bound $1 \leq \delta \leq \max \{2,1+1,0+1\}=2$. In fact, the upper bound occurs in the Drazin inverse as shown in Fig. 4. The Smith-MacMillan form $S_{(s E-A) D}^{\infty}(s)=$ $\operatorname{diag}\left(s, 1,1, \frac{1}{s}, 0\right)$ confirms that the maximum multiplicity of poles of $(s E-A)^{D}$ at $s=\infty$ is $\delta-1=1$.

\section{Conclusions}

This paper was devoted to special problems of DAEs with a singular matrix pencil. We discussed the multiplicities of poles of generalized inverses of the pencils. Our results are important for qualitative behaviour of the DAE such as solvability and uniqueness of the solution. Moreover, the discussed generalized inverses are the basis for generalized transfer functions (Hou and Müller, 1992; Hou, 1995; Hou et al., 1997). Additionally, these generalized transfer functions may also be of interest for autonomous systems. In particular, a generalized inverse of $s E-A$ can be interpreted as a transfer matrix, where the component of row $i$ and column $j$ is the transfer function of the disturbance acting on the $j$-th equation to the $i$ th variable. These properties are of interest for the perturbation index (Hairer et al., 1989). Three very simple network examples illustrate the occurrence of high index DAEs as well as singular DAEs with non-trivial Kronecker structure.

\section{Acknowledgment}

We gratefully acknowledge many helpful comments and suggestions received from several anonymous reviewers.

\section{References}

Ayasun, S., Nwankpa, C.O. and Kwatny, H.G. (2004). Computation of singular and singularity induced bifurcation points of differential-algebraic power system model, IEEE Transactions on Circuits and Systems I 51(8): 1558.

Bandler, J.W. and Zhang, Q.J. (1986). Large change sensitivity analysis in linear systems using generalized householder formulae, International Journal of Circuit Theory and Applications 14(2): 89-101.

Beelen, T. and van Dooren, P. (1988). An improved algorithm for the computation of Kronecker's canonical form of a singular pencil, Linear Algebra and Its Applications 105: 9-65.

Ben-Israel, A. and Greville, T.N.E. (1974). Generalized Inverses: Theory and Applications, Wiley-Interscience, New York, NY.

Boullion, T.L. and Odell, P.L. (1971). Generalized Inverse Matrices, Wiley-Interscience, New York, NY.

Brenan, K.E., Campbell, S.L. and Petzold, L.R. (1996). Numerical Solution of Initial-Value Problems in DifferentialAlgebraic Equations, 2nd Edn., SIAM, Philadelphia, PA.

Bunse-Gerstner, A., Byers, R., Mehrmann, V. and Nichols, N.K. (1991). Numerical computation of an analytic singular value decomposition of a matrix valued function, $\mathrm{Nu}$ merische Mathematik 60(1): 1-39.

Campbell, S.L. and Meyer, C.D. (1979). Generalized Inverses of Linear Transformations, Dover Publications, New York, NY.

Davies, A.C. (1966). Topological solution of networks containing nullators and norators, Electronics Letters 2(3): 90-92.

Demmel, J. and Kågström, B. (1993). The generalized Schur decomposition of an arbitrary pencil $\lambda A-B, A C M$ Transactions on Mathematical Software 19(2): 160-174.

Dziurla, B. and Newcomb, R. (1989). Input-output pairing in LTV semistate systems, IEEE Transactions on Circuits and Systems 36(1): 139-141.

Dziurla, B. and Newcomb, R.W. (1987). Nonregular semistate systems: Examples and input-output pairing, Proceedings of the IEEE Conference on Decision and Control, Los Angeles, CA, USA, pp. 1125-1126.

Estévez Schwarz, D. and Tischendorf, C. (2000). Structural analysis of electric circuits and consequences for MNA, International Journal of Circuit Theory and Applications 28(2): 131-162.

Fosséprez, M. (1992). Non-linear Circuits-Qualitative Analysis of Non-linear, Non-reciprocal Circuits, Wiley, Chichester.

Gantmacher, F.R. (1959). Theory of Matrices, Vol. II, Chelsea, New York, NY. 
Gear, C.W. (1971). Simultaneous numerical solution of differential-algebraic equations, IEEE Transactions on Circuit Theory 18(1): 89-95.

Gear, C.W. and Petzold, L.R. (1982). Differential/algebraic systems and matrix pencils, in B. Kågström and A. Ruhe (Eds.), Matrix Pencils, Lecture Notes in Mathematics, Vol. 973, Springer-Verlag, New York, NY, pp. 75-89.

Gear, C.W. and Petzold, L.R. (1984). ODE methods for the solution of differential/algebraic systems, SIAM Journal on Numerical Analysis 21(4): 717-728.

Griepentrog, E. and März, R. (1986). Differential-Algebraic Equations and Their Numerical Treatment, Teubner-Texte zur Mathematik, Vol. 88, Teubner Verlagsgesellschaft, Leipzig.

Günther, M. and Feldmann, U. (1999a). CAD-based electriccircuit modeling in industry, I: Mathematical structure and index of network equations, Surveys on Mathematics for Industry 8: 97-129.

Günther, M. and Feldmann, U. (1999b). CAD-based electriccircuit modeling in industry, II: Impact of circuit configurations and parameters, Surveys on Mathematics for Industry 8: $131-157$

Hairer, E., Lubich, C. and Roche, M. (1989). The Numerical Solution of Differential-Algebraic Systems by RungeKutta Methods, Lecture Notes in Mathematics, Vol. 1409, Springer-Verlag, Berlin.

Hasler, M. (1986). Non-linear non-reciprocal resistive circuits with a structurally unique solution, International Journal of Circuit Theory and Applications 14(3): 237-262.

Hayton, G.E., Pugh, A.C. and Fretwell, P. (1988). Infinite elementary divisors of a matrix polynomial and implications, International Journal of Control 47(1): 53-64.

Hou, M. (1995). Descriptor Systems: Observers and Fault Diagnosis, Fortschrittsberichte, Reihe 8: Meß-, Steuerungsund Regelungstechnik, Vol. 482, VDI Verlag, Düsseldorf.

Hou, M. and Müller, P.C. (1992). A singular matrix pencil theory for linear descriptor systems, Proceedings of the Symposium on Implicit and Nonlinear Systems, Ft Worth, TX, USA, pp. 178-190.

Hou, M., Pugh, A.C. and Hayton, G.E. (1997). Generalized transfer functions and input-output equivalence, International Journal of Control 68(5): 1163-1178.

Karcanias, N. (1987). On the characteristic, Weyr sequences, the Kronecker invariants and canonical form of a singular pencil, in R. Isermann (Ed.), Preprint from Automatic Control World Congress, Pergamon Press, Munich, pp. 109-114.

Karcanias, N. and Hayton, G.E. (1981). Generalized autonomous dynamical systems, algebraic duality and geometric theory, in H. Akashi (Ed.), Preprint from Automatic Control World Congress, Pergamon Press, Kyoto, pp. 289-294.

Kronecker, L. (1890). Algebraic reduction of pencils of bilinear forms, Sitzungsberichte der Preussischen Akademie der Wissenschaften, pp. 1225-1237, (in German).
Kublanovskaya, V.N. (1983). Analysis of singular matrix pencils, Journal of Mathematical Sciences 23(1): 1939-1950.

Kunkel, P. and Mehrmann, V. (1990). Numerical solution of differential algebraic Riccati equations, Linear Algebra and Its Applications 137/138: 39-66.

Kunkel, P. and Mehrmann, V. (2006). Differential-Algebraic Equations: Analysis and Numerical Solution, EMS Publishing House, Zürich.

Kunkel, P., Mehrmann, V., Rath, W. and Weickert, J. (1997). A new software package for linear differentialalgebraic equations, SIAM Journal on Scientific Computing 18(1): 115-138.

Kwatny, H.G., Fischl, R.F. and Nwankpa, C.O. (1995). Local bifurcation in power systems: Theory, computation, and application, Proceedings of the IEEE 83(11): 1456-1483.

Marszalek, W. and Trzaska, Z.W. (2005). Singularity-induced bifurcations in electrical power systems, IEEE Transactions on Power Systems 20(1): 312-320.

Mehrmann, V.L. (1991). The Autonomous Linear Quadratic Control Problem, Lecture Notes in Control and Information Science, Vol. 163, Springer-Verlag, Berlin.

Meyer, C.D. and Rose, N.J. (1977). The index and the Drazin inverse of block triangular matrices, SIAM Journal on Applied Mathematics 33(1): 1-7.

Müller, P.C. (2005). Remark on the solution of linear timeinvariant descriptor systems, Proceedings in Applied Mathematics and Mechanics 5(1): 175-176.

Özcaldiran, K. and Lewis, F.L. (1990). On the regularizability of singular systems, IEEE Transactions on Automatic Control 35(10): 1156-1160.

Pandolfi, L. (1981). On the regulator problem for linear degenerate control systems, Journal of Optimization Theory and Applications 33(2): 243-254.

Penrose, R. (1955). A generalized inverse for matrices, Proceedings of the Cambridge Philosophical Society 51: 406-413.

Rao, C.R. and Mitra, S.K. (1971). Generalized Inverse of Matrices and Its Applications, John Wiley \& Sons, New York, NY.

Reinschke, K. and Schwarz, P. (1976). Methods for ComputerAided Analysis of Linear Networks, Elektronisches Rechnen und Regeln, Vol. 9, Akademie-Verlag, Berlin, (in German).

Reißig, G. (1996). Differential-algebraic equations and impasse points, IEEE Transactions on Circuits and Systems I 43(3): 122-133.

Reißig, G. (1998). Contributions to the Theory and Applications of Implicit Differential Equations, Ph.D. thesis, Technische Universität Dresden, Dresden, (in German).

Reißig, G. (1999). Extension of the normal tree method, International Journal of Circuit Theory and Applications 27(2): 241-265.

Reißig, G. and Boche, H. (2003). On singularities of autonomous implicit ordinary differential equations, IEEE Transactions on Circuits and Systems I 50(7): 922-931. 
Reißig, G. and Feldmann, U. (1996). Computing the generic index of the circuit equations of linear active networks, Proceedings of the International Symposium on Circuits and Systems, Atlanta, GA, USA, Vol. III, pp. 190-193.

Riaza, R. (2004). A matrix pencil approach to the local stability analysis of non-linear circuits, International Journal of Circuit Theory and Applications 32(1): 23-46.

Riaza, R. (2006). Time-domain properties of reactive dual circuits, International Journal of Circuit Theory and Applications 34(3): 317-340.

Riaza, R. (2008). Differential-Algebraic Systems: Analytical Aspects and Circuit Applications, World Scientific, River Edge, $\mathrm{NJ}$

Röbenack, K. (1999). Contribution to the Analysis of Descriptor Systems, Shaker-Verlag, Aachen, (in German).

Röbenack, K. and Reinschke, K.J. (1998). Digraph based determination of Jordan block size structure of singular matrix pencils, Linear Algebra and Its Applications 275276: 495-507.

Röbenack, K. and Reinschke, K.J. (2000). Structural analysis of the input-output behaviour of singular descriptor systems, Workshop über Deskriptorsysteme, Paderborn, Germany, (in German).

Sannuti, P. (1981). Singular perturbations in the state space approach of linear electrical networks, International Journal of Circuit Theory and Applications 9(1): 47-57.

Sincovec, R.F., Erisman, A.M., Yip, E.L. and Epton, M.A. (1981). Analysis of descriptor systems using numerical algorithms, IEEE Transactions on Automatic Control 26(1): 139-147.

Straube, B., Reinschke, K., Vermeiren, W., Röbenack, K., Müller, B. and Clauß, C. (2001). DAE-index increase in analogue fault simulation, in R. Merker and W. Schwarz (Eds.), System Design Automation-Fundamentals, Principles, Methods, Examples, Kluwer, Boston, MA, pp. 221-232.

Tischendorf, C. (1996). Graph-theoretic determination of the structural index of algebraic-differential equations in network analysis in W. Mathis and P. Noll (Eds.), Neue Anwendungen theoretischer Konzepte in der Elektrotechnik mit Gedenksitzung zum 50. Todestag von Wilhelm Cauer, VDE-Verlag, Berlin, pp. 55-60, (in German).

Tischendorf, C. (1999). Topological index calculation of DAEs in circuit simulation, Surveys on Mathematics for Industry 8(3-4): 187-199.

van Dooren, P.M. (1981). The generalized eigenstructure problem in linear system theory, IEEE Transactions on Automatic Control 26(1): 111-129.
Vardulakis, A.I.G. and Karcanias, N. (1983). Relations between strict equivalence invariants and structure at infinity of matrix pencils, IEEE Transactions on Automatic Control 28(4): 514-516.

Vardulakis, A.I.G., Limebeer, D.N.J. and Karcanias, N. (1982) Structure and Smith-MacMillan form of a rational matrix at infinity, International Journal of Control 35(4): 701-725.

Varga, A. (1998). Computation of inner-outer factorization of rational matrices, IEEE Transactions on Automatic Control 43(5): 684-688.

Varga, A. (2001). Computing generalized inverse systems using matrix pencil methods, International Journal of Applied Mathematics and Computer Science 11(5): 1055-1068.

Weierstrass, K. (1868). On the theory of bilinear and quadratic forms, Monatsbericht der Preussischen Akademie der Wissenschaften, reprinted in Mathematische Werke von Karl Weierstrass, Vol. II, 1985, Mayer \& Müller, Berlin, pp. 310-338, (in German).

Wilkinson, J.H. (1979). Kronecker's canonical form and the QZ-algorithm, Linear Algebra and Its Applications 28 285-303.

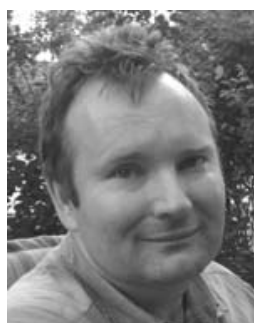

Klaus Röbenack received his Dipl.-Ing. and Dr.Ing. degrees in electrical engineering from Technische Universität Dresden in 1993 and 1999, respectively. Moreover, he received the Dipl. Math. degree in 2002 and the postdoctoral qualification (habilitation) in 2005. Prof. Röbenack has been the head of the Institute of Control Theory at the Technische Universität Dresden since 2009. His research interests include nonlinear control, observer design, descriptor systems and scientific computing

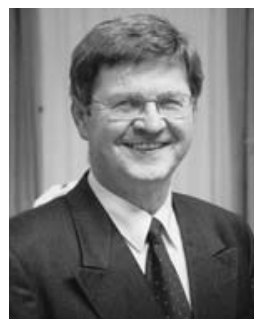

Kurt Reinschke holds doctoral degrees in applied mathematics and in electrical engineering. He was employed in industry for many years. His research has spanned a rather wide variety of topics, including computer aided circuit design, dependability theory and control methods. From 1992 to 2007, he served as a full professor and the head of the Institute of Control Theory at Technische Universität Dresden.

Received: 11 November 2009 Revised: 28 June 2010 Re-revised: 2 October 2010 